\title{
FITOSSOCIOLOGIA E ESTRUTURA DE UM FRAGMENTO FLORESTAL DA CAATINGA, CEARÁ, BRASIL
}

\author{
PHYTOSOCIOLOGY AND STRUCTURE OF A FOREST FRAGMENT CAATINGA, CEARÁ STATE, \\ BRAZIL
}

\author{
Braulio Gomes de Lima ${ }^{1}$ Maria de Fatima Barbosa Coelho²
}

\begin{abstract}
RESUMO
A maior parte do território nordestino do Brasil é ocupado por caatinga que é caracterizada por vegetação xerófita com diferentes fitofisionomias e considerada o bioma mais sensível à interferência humana e climática. Esse estudo teve o objetivo de caracterizar a composição florística e estrutura de um fragmento de caatinga localizado na Chapada Moura na Fazenda Elmo Moreno em Iguatú, Ceará. Foram estabelecidas 45 parcelas de $10 \times 20 \mathrm{~m}$ e foram medidos todos os indivíduos com diâmetro ao nível do solo $\geq 3 \mathrm{~cm}$ e altura $\geq 1 \mathrm{~m}$ Foram observados 2256 indivíduos vivos ha-1 e 165 mortos, representando 36 espécies, 29 gêneros e 13 famílias. A diversidade de espécies foi baixa em relação a outras fitofisionomias com Índice de Shannon de 1,59. Croton sonderianus e Combretum leprosum foram as espécies com maior DoR, DR, F, IVC e IVI seguidas de Pityrocarpa moniliformis. A presença de grande número de espécies lenhosas exclusivas e raras demonstra que os trabalhos realizados até o momento ainda não amostraram diversidade de espécies presentes nas diferentes regiões do Bioma. Com 70 anos de conservação, a área ainda se encontra em estágio inicial de sucessão ecológica evidenciada pela baixa riqueza florística e maior ocorrência de espécies do gênero Croton.
\end{abstract}

Palavras-chave: vegetação; espécies florestais; parâmetros fitossociológicos.

\begin{abstract}
Most of the northeastern territory of Brazil is occupied by scrub, which is characterized by xerophytic vegetation, different vegetation types and a biome considered more sensitive to human interference and climate. This study aimed to characterize the floristic composition and structure of a fragment savanna located in Chapada Moura region, at Farm Elmo Moreno Iguatu, Ceará state. Forty-five (45) plots of $10 \times 20 \mathrm{~m}$ were established and all individuals with diameter $\geq 3 \mathrm{~cm}$ and height $\geq 1 \mathrm{~m}$ were measured. Two thousand and two hundred fifty-six (2256) individuals ha ${ }^{-1}$, representing 36 species, 29 genera and 13 families were observed. Species diversity was low compared to other vegetation types with Shannon Index of 1.59. Croton sonderianus and Combretum leprosum were the species with the highest DoR, DR, F, IVC and IVI followed Pityrocarpa moniliformis. The presence of a large number of unique and rare woody species shows that the work done so far has not yet sampled the diversity of species present in the different regions of the Biome. This 70-year preserved area is still in early stages of ecological succession evidenced by low species richness and greater occurrence of species of the genus Croton.
\end{abstract}

Keywords: vegetation; forest species; phytosociological parameters.

1 Engenheiro Agronômo, Dr., Professor do Instituto Federal de Educação, Ciência e Tecnologia do Ceará, Campus Iguatu. Rodovia Iguatu,Várzea Alegre, km 05, Vila Cajazeiras, CEP 63500-000, Iguatú (CE), Brasil. braulioeafi@ hotmail.com

2 Engenheira Agronôma, Dra ${ }^{\mathrm{a}}$, Professora Titular do Programa de Pós-graduação em Agricultura Tropical da Universidade Federal de Mato Grosso, Avenida Fernando Correa da Costa, $n^{\circ}$ 2367, Cidade Universitária, CEP 78060-900, Cuiabá (MT), Brasil. Telefone/Fax +55(65) 36158618, coelhomfstrela@gmail.com

Recebido para publicação em 12/11/2013 e aceito em 9/01/2017

Ci. Fl., v. 28, n. 2, abr. - jun., 2018 


\section{INTRODUÇÃO}

A Caatinga compreende um tipo de vegetação caducifólia que cobre a maior parte da região semiárida do nordeste do Brasil, e se espalha por uma área de cerca de $800.000 \mathrm{~km}^{2}$. A vegetação de caatinga tem várias fitofisionomias cuja distribuição é determinada em grande parte pelo clima, topografia e geologia e em suas múltiplas inter-relações resultam em diferentes ambientes ecológicos (RODAL; MARTINS; SAMPAIO, 2008).

A complexidade e diversidade desta região são ampliadas, pois é a única ecorregião de floresta seca tropical do mundo, rodeada por florestas e úmidas (BRASIL, 2006). Estudos sobre a composição e estrutura da caatinga são importantes para a caracterização das diferentes fácies, constituindo ferramenta para entender aspectos da ecologia regional, fornecendo bases para a sua conservação sustentável (GUEDES et al., 2012).

No entanto, entre os biomas brasileiros, a Caatinga é provavelmente o mais subestimado e pouco conhecido botanicamente. Apenas 7,5\% do território da caatinga estão protegidos em áreas de conservação e 1,4\% dessas reservas são estritamente áreas protegidas (TOLEDO, 2013). Esta situação é resultado da crença injustificada de que a vegetação da caatinga é o resultado da modificação de outra formação vegetal e está associada com uma baixa diversidade de plantas sem espécies endêmicas e altamente modificada pelas ações humanas (GIULIETTI et al., 2002).

Os estudos sobre a caatinga tem sido ampliados em diversos trabalhos (PEGADO et al., 2006; QUEIROZ et al., 2006; SANTANA; SOUTO, 2006; PINHEIRO; ALVES, 2007; PESSOA et al., 2008; RODAL; MARTINS; SAMPAIO, 2008; RODAL; COSTA; SILVA, 2008; ANDRADE et al., 2009; RAMALHO et al., 2009; SOUZA; RODAL, 2010; BESSA; MEDEIROS, 2011; ARAÚJO et al., 2012; BARBOSA et al., 2012; GUEDES et al., 2012; SANTOS; JERONIMO, 2013; SOUZA; MEDEIROS, 2013).

Nesses estudos verifica-se que a flora da depressão sertaneja (caatinga sensu stricto) é distinta da vegetação que ocorre nos maciços/serras, onde há registro de formações florestais com diferentes níveis de caducifolia. A caatinga sensu stricto é o tipo vegetacional mais estudado, entretanto, outra unidade que tem destaque por sua extensão é a área sedimentar, onde a flora é distinta do embasamento cristalino, tanto da depressão sertaneja (ARAÚJO; SAMPAIO; RODAL, 1995) como dos maciços/serras cristalinos (RODAL; NASCIMENTO, 2006).

A escolha do fragmento florestal em Iguatu-CE para desenvolver este estudo deve-se ao estágio de preservação da vegetação, possível garantia de preservação futura e também por ser uma área sedimentar. Assim, o presente trabalho visa estudar a composição florística e a estrutura fitossociológica dessa formação vegetal para contribuir para o conhecimento sobre a vegetação em áreas sedimentares da caatinga e também para subsidiar o manejo, regeneração, conservação e preservação.

\section{MATERIAL E MÉTODO}

\section{Área de estudo}

A região de estudo está localizada no município de Iguatu, Centro Sul do Estado do Ceará, com $1.042,6 \mathrm{~km}^{2}$, altitude $213 \mathrm{~m}$, e localiza-se a $400 \mathrm{~km}$ da capital Fortaleza. O relevo do município é constituído por extensas várzeas planas e a vegetação existente compõe-se de caatinga, em sua maioria arbustiva densa. O clima é tropical quente semiárido, a estação chuvosa compreende os meses de janeiro a maio/junho. A precipitação média anual é de 807 milímetros, , especialmente o mês de abril é o mais chuvoso. A média da temperatura máxima anual e mínima são 32 e $26^{\circ} \mathrm{C}$, respectivamente. A umidade relativa anual chega a uma média de $61,8 \%$, enquanto a taxa média de evaporação chega a 2.248 milímetros/ano. A insolação média $236 \mathrm{~h} /$ mês e a velocidade média do vento é de $1,8 \mathrm{~m} / \mathrm{s}$, com predominância para o leste-sudeste (INSTITUTO DE PLANEJAMENTO DO CEARÁ, 2012).

A pesquisa foi conduzida em um fragmento florestal, na Chapada Moura, localizado na Fazenda 
Elmo Moreno - 6²1'78,3"S $/ 39^{\circ} 14^{\prime} 23,6^{\prime \prime} \mathrm{W}$. A área é preservada, apesar de alguns sinais da exploração sustentável de espécies madeireiras de maior utilidade econômica regional, como Mimosa tenuiflora (Willd.) Poir., Mimosa caesalpiniifolia Benth., Myracrodruon urundeuva Allemão, Anadenanthera colubrina var. cebil (Griseb.) Altschul, Croton sonderianus Müll. Arg. e Amburana cearensis (Allemão). A.C.Sm. Um fato relevante é que não há registro da retirada total da vegetação nos últimos 70 anos, como comprovou a inspeção visual e informações obtidas a partir dos antigos moradores na região. O substrato da propriedade e área de estudo é sedimentar, parte da bacia sedimentar de Iguatu. O solo é do tipo o Latossolo, com horizonte B latossólico, não hidromórfico, sendo mais normais as transições difusas e graduais entre os horizontes; predominantemente profundo a muito profundo, muito poroso e muito friável quando úmido e fortemente drenado (EMBRAPA, 2006).

\section{Coleta e tratamento dos dados}

O levantamento fitossociológico foi realizado no período de agosto 2008 a julho de 2010. Foram sorteadas 45 parcelas por meio de marcação aleatória de coordenadas geográficas obtidas com o auxílio do software GPS TrackMaker ${ }^{\circledR}$ (FERREIRA JUNIOR, 2010), as quais foram transferidas para um aparelho GPS para a localização em campo.

No levantamento do componente lenhoso foi utilizado o método de estabilização da curva do coletor, utilizando parcelas com dimensões de $10 \mathrm{~m}$ x $20 \mathrm{~m}$, método este comumente utilizado no nordeste em estudos quantitativos de caatinga e do carrasco (RODAL; SAMPAIO; FIGUEIREDO, 2013). Foram amostrados os indivíduos vivos e mortos, ainda em pé, excluídos os cipós e bromeliáceas, que se individualizaram ao nível do solo e que atendiam ao critério de inclusão, ou seja, altura total maior ou igual a $1 \mathrm{~m}$ e diâmetro do caule ao nível do solo maior ou igual a $3 \mathrm{~cm}$. Em cada parcela foram medidas as alturas totais e diâmetro do caule na base dos indivíduos que atendiam ao critério e se individualizavam ao nível do solo. As medidas do diâmetro do caule a altura do solo foram tomadas com paquímetro (até o diâmetro de $15 \mathrm{~cm}$ ), acima disto foram tomados os perímetros com fita métrica e as alturas com uma régua de cano PVC.

Com os dados quantitativos obtidos, analisou-se a estrutura de abundância e de tamanho, calcularamse os parâmetros relativos de densidade, frequência e dominância, o valor de importância (VI), valor de cobertura (VC) relativa por espécie e os índices de diversidade de Shannon (H') e de equitabilidade (J) de acordo com Mueller-Dombois e Ellenberg (1974) e Magguran (1989).

\section{RESULTADOS E DISCUSSÃO}

A curva espécie-área (Figura 1) ajusta-se a uma equação polinomial com coeficiente de determinação significativo $\left(\mathrm{R}^{2}=0,98\right)$. A estabilização ocorreu com 24 parcelas e 36 espécies acumuladas.

A amostragem para a representação da variabilidade florística foi satisfatória, uma vez que se observa uma tendência à estabilidade (inflexão da curva), a partir da amostragem de uma área de $4.200 \mathrm{~m}^{2}$. Guedes et al. (2012) estudando um trecho de caatinga da Paraíba, com parcelas de 20x20 m que ocuparam $4.000 \mathrm{~m}^{2}$, verificaram estabilização da curva na oitava parcela.

Foram amostrados 2.421 indivíduos, o que corresponde à densidade estimada de 5.043,8 indivíduos ha $^{-1}$, sendo $2.256(93,2 \%)$ indivíduos vivos e $165(6,8 \%)$ mortos ainda em pé. A área basal total $\left(13,71 \mathrm{~m}^{2}\right)$, incluídos os indivíduos vivos e mortos ainda em pé, correspondeu a $28,56 \mathrm{~m}^{2} \mathrm{ha}^{-1}$. A densidade constatada na área experimental estudada é considerada elevada quando comparada com resultados obtidos por Rodal, Costa e Silva (2008), densidade de 2.172 indivíduos ha ${ }^{-1} \mathrm{em}$ área de caatinga arbustivo-arbórea aberta, substrato cristalino e por Cestaro e Soares (2004) que encontraram densidade de 1.924 indivíduos ha $^{-1}$ em área de caatinga antropizada, substrato sedimentar.

A elevada densidade do fragmento florestal estudado pode ser explicada como um reflexo da baixa ação antropogênica no decorrer dos últimos 70 anos, refletindo em uma maior conservação do remanescente florestal. 


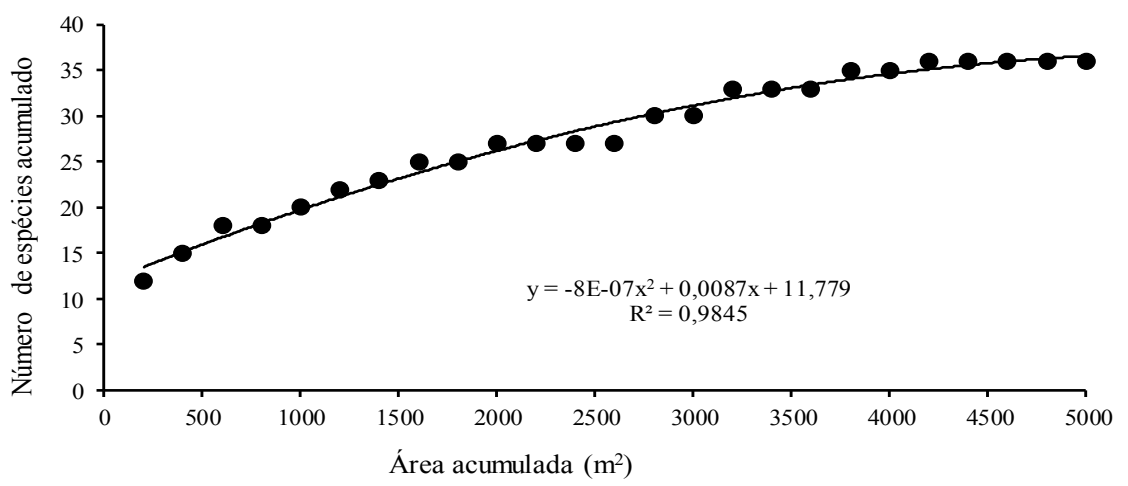

FIGURA 1: Curva de estabilização das espécies na área da Chapada Moura - Fazenda Elmo Moreno, Iguatú, Ceará.

FIGURE 1: Curve stabilization of the species in the area of Chapada Moura - Farm Elmo Moreno, Iguatu, Ceará state.

A área basal total dos indivíduos classificados no estrato arbustivo-arbóreo foi superior aos valores encontrados por Santana e Souto (2006) $\left(17,50 \mathrm{~m}^{-2}\right.$. ha-1). Porém, o valor foi o mesmo encontrado por Pereira Junior, Andrade e Araújo (2012) $\left(28,76 \mathrm{~m}^{-2} . \mathrm{ha}^{-1}\right)$ estudando uma área de caatinga na Paraíba.

O componente lenhoso da área da Chapada Moura está representado por 36 espécies, 29 gêneros e 13 famílias. Os valores dos parâmetros fitossociológicos relativos de dominância, densidade e frequência, o valor de cobertura e o valor de importância podem ser analisados na Tabela 1.

TABELA 1: Parâmetros fitossociológicos das espécies representadas no componente lenhoso amostradas na área da Chapada Moura - Fazenda Elmo Moreno, Iguatú, Ceará. DoR = dominância relativa; DR = densidade relativa; $\mathrm{FR}=$ frequência relativa; $\mathrm{VC}=$ valor de cobertura; $\mathrm{VI}=$ valor de importância.

TABLE 1: Parameters phytosociological species represented in the woody component in the sampled area of Chapada Moura - Farm Elmo Moreno, Iguatu, Ceará state. DoR $=$ relative dominance, $\mathrm{DR}=$ relative density, relative frequency $=\mathrm{FR} ; \mathrm{VC}=$ coverage value; $\mathrm{VI}=$ importance value.

\begin{tabular}{|c|c|c|c|c|c|c|}
\hline Espécies & Famílas & DoR & DR & FR & $\mathrm{VC}$ & VI \\
\hline Croton sonderianus Müll.Arg. & Euphorbiaceae & 10,36 & 58,36 & 11,59 & 68,72 & 80,32 \\
\hline Combretum leprosum Mart. & Combretaceae & 11,40 & 19,74 & 11,59 & 31,15 & 42,74 \\
\hline Pityrocarpa moniliformis (Benth.) Luckow \& & Fabaceae-Mimosoideae & & & & & \\
\hline R.W.Jobson & & 14,79 & 3,470 & 9,18 & 18,26 & 27,44 \\
\hline Piptadenia stipulacea (Benth.) Ducke & Fabaceae-Mimosoideae & 8,46 & 2,89 & 8,70 & 11,36 & 20,05 \\
\hline Poincianella bracteosa (Tul.) L.P.Queiroz & Fabaceae-Caesalpinioideae & 6,39 & 0,83 & 6,28 & 7,22 & 13,50 \\
\hline Senegalia tenuifolia (L.) Britton \& Rose & Fabaceae-Mimosoideae & 3,80 & 1,69 & 5,80 & 5,49 & 11,29 \\
\hline Mimosa caesalpiniifolia Benth. & Fabaceae-Mimosoideae & 6,48 & 2,81 & 5,31 & 9,29 & 14,60 \\
\hline Mimosa tenuiflora (Willd.) Poir. & Fabaceae-Mimosoideae & 3,37 & 1,20 & 4,83 & 4,56 & 9,40 \\
\hline Senna trachypus (Benth.) H.S.Irwin \& Barneby & Fabaceae-Caesalpinioideae & 2,74 & 1,94 & 4,35 & 4,68 & 9,03 \\
\hline Helicteres muscosa Mart. & Malvaceae & 2,06 & 0,58 & 4,35 & 2,63 & 6,98 \\
\hline Amburana cearensis (Allemão) A.C.Sm. & Fabaceae-Faboideae & 5,74 & 0,45 & 3,38 & 6,19 & 9,57 \\
\hline Mimosa arenosa (Willd.) Poir. & Fabaceae-Mimosoideae & 3,56 & 1,69 & 3,38 & 5,26 & 8,64 \\
\hline Senegalia polyphylla (DC.) Britton \& Rose & Fabaceae-Mimosoideae & 3,06 & 0,33 & 2,42 & 3,39 & 5,81 \\
\hline Combretum glaucocarpum Mart. & Combretaceae & 1,81 & 0,74 & 1,93 & 2,55 & 4,49 \\
\hline Anadenanthera colubrina var. cebil (Griseb.) & Fabaceae-Mimosoideae & & & & & \\
\hline Altschul & & 2,18 & 0,17 & 1,45 & 2,35 & 3,80 \\
\hline $\begin{array}{l}\text { Libidibia ferrea (Mart. ex Tul.) L.P.Queiroz var. } \\
\text { ferrea }\end{array}$ & Fabaceae-Caesalpinioideae & 139 & & & 151 & 296 \\
\hline Cynophalla flexuosa (L.) J.Presl & Capparaceae & $\begin{array}{l}1,39 \\
1,01\end{array}$ & 0,21 & $\begin{array}{l}1,45 \\
1,45\end{array}$ & $\begin{array}{l}1,21 \\
1,21\end{array}$ & 2,66 \\
\hline Pseudobombax marginatum (A.St.-Hil.) A. Robyns & Malvaceae & 1,06 & 0,12 & 1,45 & 1,19 & 2,64 \\
\hline
\end{tabular}


TABELA 1: Continuação...

TABLE 1: Continued...

\begin{tabular}{|c|c|c|c|c|c|c|}
\hline Espécies & Famílas & DoR & DR & FR & $\mathrm{VC}$ & VI \\
\hline Aspidosperma pyrifolium Mart. & Apocynaceae & 0,90 & 0,74 & 0,97 & 1,64 & 2,61 \\
\hline Ximenia americana $\mathrm{L}$. & Olacaceae & 0,69 & 0,70 & 0,97 & 1,39 & 2,36 \\
\hline Bauhinia cheilantha (Bong.) Steud. & Fabaceae-Caesalpinioideae & 1,01 & 0,21 & 0,97 & 1,22 & 2,19 \\
\hline Bauhinia pentandra (Bong.) D.Dietr. & Fabaceae-Caesalpinioideae & 0,99 & 0,08 & 0,97 & 1,08 & 2,04 \\
\hline Não ident. 1 & Myrtaceae & 0,58 & 0,12 & 0,97 & 0,70 & 1,67 \\
\hline Handroanthus impetiginosus (Mart. ex DC.) & Bignoniaceae & & & & & \\
\hline Mattos & & 0,87 & 0,04 & 0,48 & 0,92 & 1,40 \\
\hline Adenocalymna apparicianum J.C.Gomes & Bignoniaceae & 0,75 & 0,04 & 0,48 & 0,80 & 1,28 \\
\hline Myracrodruon urundeuva Allemão & Anacardiaceae & 0,62 & 0,12 & 0,48 & 0,74 & 1,22 \\
\hline Helicteres baruensis Jacq. & Malvaceae & 0,67 & 0,04 & 0,48 & 0,71 & 1,19 \\
\hline Chloroleucon dumosum (Benth.) G.P.Lewis & Fabaceae-Mimosoideae & 0,56 & 0,12 & 0,48 & 0,68 & 1,16 \\
\hline Cochlospermum vitifolium (Willd.) Spreng. & Bixaceae & 0,60 & 0,04 & 0,48 & 0,64 & 1,12 \\
\hline Manihot carthaginensis subsp. glaziovii & Euphorbiaceae & & & & & \\
\hline (Müll.Arg.) Allem & & 0,47 & 0,08 & 0,48 & 0,55 & 1,03 \\
\hline Jatropha mollissima (Pohl) Baill. & Euphorbiaceae & 0,48 & 0,04 & 0,48 & 0,52 & 1,00 \\
\hline Cordia trichotoma (Vell.) Arráb. ex Steud. & Boraginaceae & 0,36 & 0,04 & 0,48 & 0,40 & 0,88 \\
\hline Cereus jamacaru DC. & Cactaceae & 0,26 & 0,04 & 0,48 & 0,30 & 0,79 \\
\hline Myriopus rubicundus (Salzm. ex DC.) Luebert & Boraginaceae & 0,18 & 0,08 & 0,48 & 0,26 & 0,74 \\
\hline Machaerium acutifolium Vogel & Fabaceae-Faboideae & 0,20 & 0,04 & 0,48 & 0,24 & 0,72 \\
\hline Totais & & 100,0 & 100,0 & 100,0 & 200,0 & 300,0 \\
\hline
\end{tabular}

O número de espécies observadas nesse trabalho (36) encontra-se dentro da faixa observada em outros trabalhos desenvolvidos em regiões semiáridas (LEMOS; RODAL, 2002; ALCOFORADO-FILHO; SAMPAIO; RODAL, 2003; PEGADO et al., 2006; QUEIROZ et al., 2006; SANTANA; SOUTO, 2006; PINHEIRO; ALVES, 2007; PESSOA et al., 2008; RODAL; MARTINS; SAMPAIO, 2008; RODAL; COSTA; SILVA, 2008; ANDRADE et al., 2009; RAMALHO et al., 2009; SOUZA; RODAL, 2010; BESSA; MEDEIROS, 2011; ARAÚJO et al., 2012; BARBOSA et al., 2012; GUEDES et al., 2012; SANTOS; JERONIMO, 2013; SOUZA; MEDEIROS, 2013), os quais constataram um número variando entre 35 e 96 espécies para áreas de caatinga. O maior ou menor número de espécies nos levantamentos realizados deve ser resposta a um conjunto de fatores, tais como situação topográfica, classe, profundidade e permeabilidade do solo e não apenas quantidade de chuvas, embora este seja um dos fatores mais importantes.

Guedes et al. (2012) trabalhando com um menor número de indivíduos arbóreos (649) encontraram menor número de espécies: 21 espécies distribuídas em 21 gêneros e 11 famílias botânicas enquanto Pereira Junior, Andrade e Araújo (2012) em um levantamento de 3.495 indivíduos encontraram 14 famílias, 26 gêneros e 37 espécies.

Pityrocarpa moniliformis apresentou a maior dominância relativa (14,8\%), seguida de Combretum leprosum $(11,40 \%)$ e Croton sonderianus $(10,36 \%)$. As duas últimas espécies, apesar de apresentarem maior número de indivíduos que Pityrocarpa moniliformis, perdem na dominância em virtude de apresentar indivíduos com menores diâmetros. Combretum leprosum e Croton sonderianus são espécies do grupo das pioneiras, e a área está em recuperação. Estes resultados corroboram com Pereira Junior, Andrade e Araújo (2012).

As espécies Croton sonderianus e Combretum leprosum apresentaram densidade relativa bem superior às demais espécies e contribuíram com $78,0 \%$ do total de indivíduos. Croton sonderianus contribuiu com 58,3\% do número total de indivíduos amostrados, seguido por Combretum leprosum com 19,7\%. A maioria (75\%) das espécies contribuiu com menos de $1 \%$ da densidade relativa. Entre estas, Helicteres muscosa, com população considerável nesta área de substrato sedimentar, amostrada com um pequeno número de indivíduos devido ao critério de inclusão, que selecionou apenas os indivíduos de porte estabelecido. Estas espécies são pioneiras de áreas de florestas secundárias, o que indica que a área ainda está em recuperação.

As espécies Cereus jamacaru, Cochlospermum vitifolium, Cordia trichotoma, Helicteres baruensis, Jatropha mollissima, Machaerium acutifolium e Handroanthus impetiginosus, com poucos indivíduos na comunidade, podem ser consideradas raras; são espécies que não seriam amostrados em maior número, 
mesmo mudando o critério de inclusão. Essas espécies foram amostradas com um só indivíduo, indicando que sua densidade média seria próxima a dois indivíduos por hectare. As espécies Bauhinia pentandra, Manihot carthaginensis spbsp. Glaziovii e Myriopus rubicundus foram representadas por dois indivíduos. Oito espécies foram amostradas em 3 a 5 indivíduos (Bauhinia cheilantha, Cynophalla flexuosa, Anadenanthera colubrina var. cebil, Libidibia ferrea var. ferrea, Chloroleucon dumosum. Myracrodruon urundeuva, Pseudobombax marginatum e uma Myrtaeae não identificada). As demais espécies foram representadas por pelo menos oito indivíduos.

Quanto ao valor de importância (VI) verificou-se que mais da metade $(56,8 \%)$ concentrou-se em apenas quatro (11,1\% do total amostrado) espécies: Croton sonderianus, Combretum leprosum, Pityrocarpa moniliformis e Piptadenia stipulacea. Essas espécies juntamente com Poincianella bracteosa, Senegalia tenuifolia, Mimosa caesalpiniifolia, Mimosa tenuiflora e Senna trachypus representam as nove espécies que somam 76,1\% do VI de todas as espécies amostradas (25\% do total amostrado) (Figura 2). As famílias Fabaceae, Euphorbiaceae e Combretaceae representam 90,6\% do VI total da área amostrada.

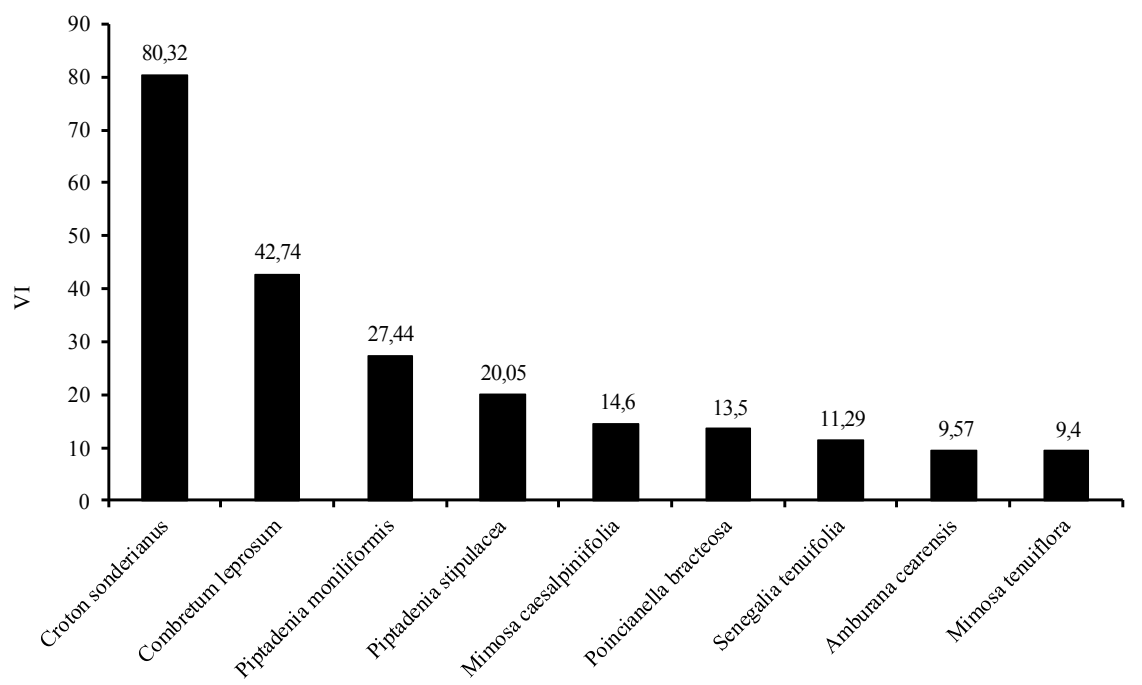

FIGURA 2: Espécies com os maiores valores de importância (VI) encontradas na área da Chapada Moura - Fazenda Elmo Moreno, Iguatú, Ceará.

FIGURE 2: Species with the highest importance value (VI) found in the area of Chapada Moura - Farm Elmo Moreno, Iguatu, Ceará state.

A maior dominância relativa das espécies Pityrocarpa moniliformis (14,8\%), seguida de Combretum leprosum $(11,40 \%)$ e Croton sonderianus $(10,36 \%)$ tem sido verificada em outros trabalhos na caatinga. Em estudo conduzido na Serra do Mel - RN, Cezar et al. (2006) também verificaram maior dominância de Pityrocarpa moniliformis em relação a outras espécies, provavelmente devido à grande facilidade de rebrota dessa espécie, e este fenômeno também é observado em Combretum leprosum e Croton sonderianus.

A família Euphorbiaceae com maior VI dentre as famílias na área estudada é frequentemente citada entre as que possuem maior riqueza de espécies em diferentes áreas de caatinga do Nordeste brasileiro (ARAÚJO; SAMPAIO; RODAL, 1995; FERRAZ et al., 1998; ALCOFORADO-FILHO; SAMPAIO; RODAL, 2003; LEMOS; MEGURO, 2010).

As espécies Machaerium acutifolium, Pityrocarpa moniliformis e Combretum glaucocarpum foram registradas também no cerrado do Piauí (CASTRO; MARTINS; FERNANDES, 1998). Libidibia ferrea, Pityrocarpa moniliformis e Piptadenia stipulacea foram também amostradas em caatinga, carrasco, cerrado e floresta seca por Lemos e Meguro (2010). Quanto às espécies Aspidosperma pyrifolium, Libidibia ferrea, Handroanthus impetiginosus e Dioclea grandiflora foram registradas em levantamentos realizados no semiárido nordestino, em áreas categorizadas como formação decidual (LEMOS; MEGURO, 2010).

Com relação às características estruturais, cinco espécies (13,8\%) apresentaram diâmetros médios acima de $15 \mathrm{~cm}$, ou seja, maior área média basal, dentre elas Amburana cearensis, Aspidosperma pyrifolium, Mimosa caesalpiniifolia, Pityrocarpa moniliformis e Handroanthus impetiginosus e $52 \%$ diâmetro 
maior que $6 \mathrm{~cm}$ (Figura 3A) indicando que a área está conservada com pouca antropização.

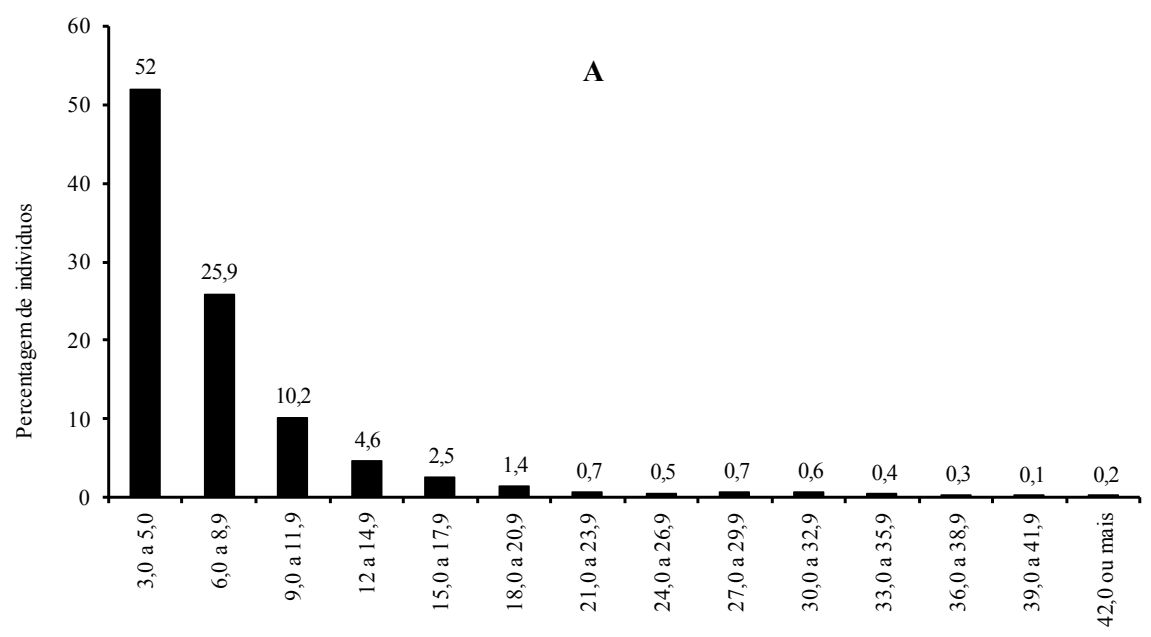

Classes de diametro

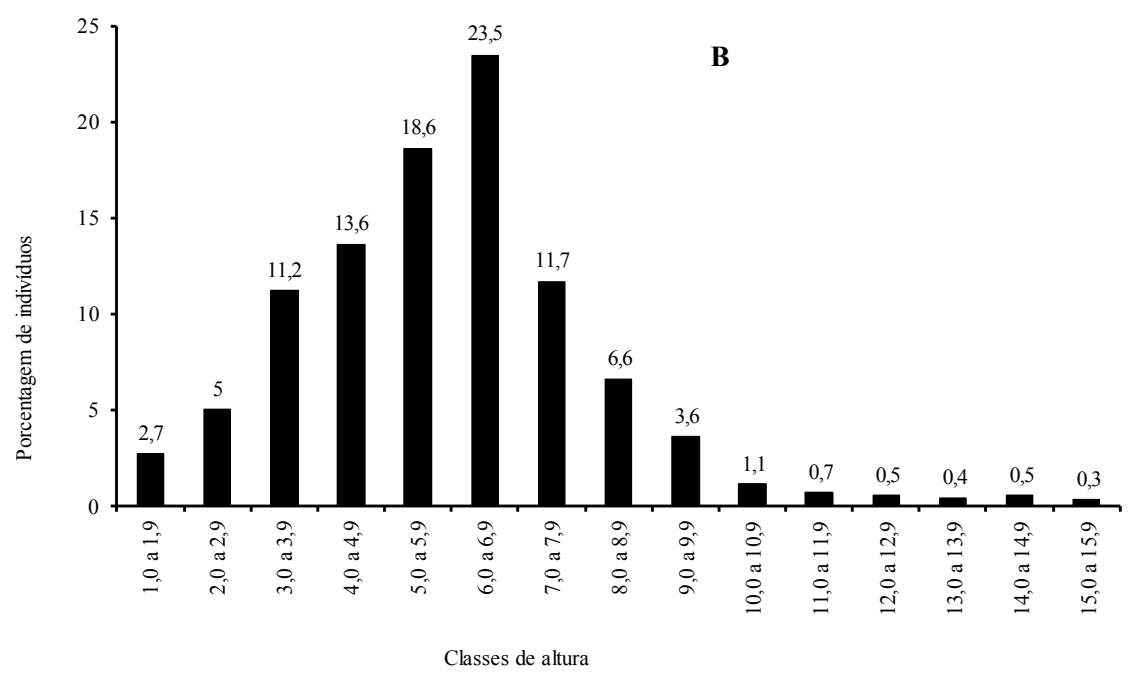

FIGURA 3: Percentual de indivíduos (\%) por classes de diâmetro (A) e de altura (B) das espécies do componente lenhoso amostradas na área da Chapada Moura - Fazenda Elmo Moreno, Iguatú, Ceará. Diâmetro médio $=7,4 \mathrm{~cm}$; Desvio padrão $\mathrm{s}=6,0 \mathrm{~cm}$; amplitude $=2,0$ a $84,7 \mathrm{~cm}$. Altura média $=5,8 \mathrm{~m}$; Desvio padrão $\mathrm{s}=2,2 \mathrm{~m}$; amplitude $=1,0$ a $15,6 \mathrm{~m}$.

FIGURE 3: Percentage of individuals (\%) by diameter classes (A) and height (B) of the woody component species sampled in the area of Chapada Moura - Farm Elmo Moreno, Iguatu, Ceará state. Mean diameter = $7.4 \mathrm{~cm}$; standard deviation s $=6.0 \mathrm{~cm}$; amplitude $=2.0$ to $84.7 \mathrm{~cm}$. Average height $=5.8 \mathrm{~m}$; standard deviation $\mathrm{s}=2.2 \mathrm{~m}$, range $=1.0$ to $15.6 \mathrm{~m}$.

Dezessete espécies (47,2\% do total amostrado) apresentaram diâmetros máximos acima de $20 \mathrm{~cm}$, como Mimosa caesalpiniifolia $(78,6 \mathrm{~cm})$, Pityrocarpa moniliformis $(56,3 \mathrm{~cm})$, Amburana cearensis $(40,4$ $\mathrm{cm})$, Handroanthus impetiginosus $(39,8 \mathrm{~cm})$, Aspidosperma pyrifolium $(31,2 \mathrm{~cm})$ e Croton sonderianus $(34,5 \mathrm{~cm})$.

Quanto à altura total verificou-se que 32 espécies $(88,8 \%$ do total) apresentaram indivíduos com alturas superiores a $6 \mathrm{~m}$ (48,9\% do total de indivíduos) (Figura 3B). Amburana cearensis, Anadenanthera colubrina, Poincianella bracteosa, Combretum leprosum, Croton sonderianus, Mimosa caesalpiniifolia, Mimosa tenuiflora, Pityrocarpa moniliformis, Senegalia pollyphylla e Handroanthus impetiginosus, fazem parte deste grupo. Esse grupo de espécies apresenta indivíduos com alturas bem superiores à média geral (5,8 m) dos elementos arbóreos que compõem a área. As espécies Pityrocarpa moniliformis, Amburana 
cearensis, Anadenanthera colubrina e Poincianella bracteosa apresentaram alturas entre 13 e $16 \mathrm{~m}$. No entanto, a distribuição de frequência das alturas dos indivíduos arbóreos indica maior concentração $(42,1 \%)$ entre 5,0 a 6,9 m (Figura 3B).

A área estudada apresenta baixa diversidade de espécies se comparada com outras formações vegetacionais, com o índice de Shannon $\mathrm{H}=1,59$ nats, mas este valor é equivalente ao encontrado em outras regiões da caatinga (FERRAZ; ALBUQUERQUE; MEUNIER, 2006; RODAL; NASCIMENTO, 2006; PESSOA et al., 2008; OLIVEIRA et al. 2009; GUEDES et al., 2012; MARANGON et al., 2013). O valor da equabilidade, constatada nesse estudo, também apresentou um valor baixo $(\mathrm{J}=0,53)$, quando comparado com os valores encontrados por Pegado et al. (2006) que, estudando o estrato arbustivo-arbóreo da caatinga no município de Monteiro-PB, encontraram valores elevados para esse parâmetro $(J=0,73$ e 0,79$)$, porém, é semelhante aos constatados por Oliveira et al. (2009) e Pereira Junior, Andrade e Araújo (2012), estudando vegetações em regiões semiáridas.

Com relação à mortalidade dos indivíduos (Tabela 2) os valores levantam questões sobre o grau de preservação dessas áreas, formas e estratégias ecofisiológicas desenvolvidas pelas espécies, longevidade e antropismo. As espécies Senna trachyphus e Mimosa tenuiflora apresentaram altos índices de indivíduos mortos, isto ocorrendo principalmente nas parcelas em que há maior competição de espécies, especialmente se forem sombreadas. Entretanto, sabe-se que Mimosa tenuiflora é intensamente explorada na caatinga para a confecção de carvão. A espécie Combretum leprosum, que detém o segundo VI na área, apresentou 10 indivíduos mortos $(2,0 \%$, índice relacionado à longevidade dessa espécie e por ser pioneira de formações florestais em sucessão secundária.

TABELA 2: Número total de indivíduos, número de mortos e percentagem por espécie com mais de cinco indivíduos na área da Chapada Moura, Fazenda Elmo Moreno, Iguatu, Ceará.

TABLE 2: Total number of individuals, number of deaths and percentage by species with more than five individuals in the area of Chapada Moura, Farm Elmo Moreno, Iguatu, Ceará state.

\begin{tabular}{|c|c|c|c|}
\hline Espécies & $\mathrm{N}^{\circ}$ de indivíduos & $\mathrm{N}^{\circ}$ de mortos & $\%$ de mortos \\
\hline Croton sonderianus & 1412 & 44 & 3,1 \\
\hline Combretum leprosum & 478 & 10 & 2,1 \\
\hline Pityrocarpa moniliformis & 84 & 8 & 9,5 \\
\hline Piptadenia stipulacea & 70 & 10 & 14,3 \\
\hline Mimosa caesalpiniifolia & 68 & 15 & 22,1 \\
\hline Senna trachyphus & 47 & 37 & 78,7 \\
\hline Senegalia tenuiflora & 41 & 2 & 4,9 \\
\hline Mimosa arenosa & 41 & 2 & 4,9 \\
\hline Mimosa tenuiflora & 29 & 17 & 58,6 \\
\hline Poincianella bracteosa & 20 & 1 & 5,0 \\
\hline Aspidosperma pyrifolium & 18 & 1 & 5,6 \\
\hline Helicteres muscosa & 14 & 2 & 14,3 \\
\hline Total & 2322 & 149 & 6,5 \\
\hline
\end{tabular}

O conjunto de espécies que compõe a área apresenta uma tendência maior para a província da caatinga, que Veloso, Rangel-Filho e Lima (1991) consideraram caatinga strito sensu, não sendo área ecotonal decidual, de transição entre carrasco e cerrado. Apresenta dominância de algumas espécies como Croton sonderianus e Mimosa tenuiflora, típicas da caatinga (ANDRADE-LIMA, 1989; LEWIS, 1998), além de Combretum leprosum, Pityrocarpa moniliformis e Mimosa caesalpiniifolia, que se distribuem em vegetação de carrasco e cerrado. A presença de cactáceas é rara, ocorrendo apenas Cereus jamacaru DC. e não há ocorrência de Bromelia laciniosa Mart. ex Schult. \& Schult.f.

\section{CONCLUSÕES}

Este trabalho destaca a caatinga da Chapada Moura como possuidora de baixa riqueza florística com 36 espécies arbóreas pertencentes a 21 famílias. O componente não lenhoso foi mais representativo com $62,2 \%$ das espécies amostradas. Croton sonderianus e Combretum leprosum foram as espécies com maior DoR, DR, F, VC e VI seguidas de Pityrocarpa moniliformis. O registro de um significativo número de espécies vegetais lenhosas exclusivas e raras demonstra que os trabalhos realizados até o momento ainda 
não amostraram considerável número de espécies presentes nas diferentes regiões do bioma Caatinga. Com 70 anos de conservação, a área ainda se encontra em estágio inicial de sucessão ecológica evidenciada pela baixa riqueza florística e pela maior ocorrência de espécies do gênero Croton que são pioneiras de áreas de florestas secundárias.

\section{AGRADECIMENTOS}

Ao Banco do Nordeste - BNB pela concessão de auxilio e ao Conselho Nacional de Desenvolvimento Científico e Tecnológico - CNPq pela concessão de Bolsa de Produtividade.

\section{REFERÊNCIAS}

ALCOFORADO-FILHO, F. G.; SAMPAIO, E. V. S. B.; RODAL, M. J. N. Florística e fitossociologia de um remanescente de vegetação caducifólia espinhosa arbórea em Caruaru, Pernambuco. Acta Botanica Brasílica, Feira de Santana, v. 17, n. 2, p. 287-303, 2003.

ANDRADE, M. V. M. et al. Levantamento florístico e estrutura fitossociológica do estrato herbáceo e subarbustivo em áreas de caatinga no Cariri paraibano. Revista Caatinga, Mossoró, v. 22, n. 1, p. 229-237, 2009.

ANDRADE-LIMA, D. Plantas das caatingas. Rio de Janeiro: Academia Brasileira de Ciências, 1989. 243 p.

ARAÚJO, B. A. et al. Estrutura fitossociológica em uma área de caatinga no seridó paraibano. Revista Educação Agrícola Superior, Brasília, v. 27, n. 1, p. 25-29, 2012.

ARAÚJO, E. L.; SAMPAIO, E. V. S. B.; RODAL, M. J. N. Composição florística e fitossociológica de três áreas de Caatinga de Pernambuco. Revista Brasileira de Biologia, São Carlos, v. 55, n. 4, p. 595-607, 1995.

BARBOSA, M. D. et al. Florística e fitossociologia de espécies arbóreas e arbustivas em uma área de caatinga em Arcoverde, PE, Brasil. Revista Árvore, Viçosa, MG, v. 36, n. 5, p. 851-858, 2012.

BESSA, M. A. P.; MEDEIROS, J. F. Levantamento florístico e fitossociológico em fragmentos de caatinga no município de Taboleiro Grande-RN. Geotemas, Pau dos Ferros, v. 1, n. 2, p. 69-83, 2011.

BRASIL. Ministério do Meio Ambiente. Secretaria de Biodiversidade e Florestas. Conservação e uso sustentável em áreas protegidas e corredores: uma contribuição para a superação da pobreza nos biomas caatinga e cerrado. Brasília: MMA, 2006. 38 p.

CASTRO, A. A. J. F.; MARTINS, F. R.; FERNANDES, A. G. The woody flora of cerrado vegetation in the state of Piauí, Northeastern Brazil. Edinburgh Journal of Botany, Edinburgh, v. 55, p. 455-472, 1998.

CESTARO, L. A.; SOARES, J. Variações florística e estrutural e relações fitogeográficas de um fragmento de floresta decídua no Rio Grande do Norte, Brasil. Acta Botanica Brasilica, Feira de Santana, v. 18, n. 2, p. 203-218, 2004.

CEZAR, A. F. et al. Flora da Serra do Mel na Vila Alagoas. Revista Verde de Agroecologia, Mossoró, v. 1, n. 2, p. 100-112, 2006.

EMBRAPA. Sistema Brasileiro de Ciência dos Solos - SiBCs. 2. ed. Rio de Janeiro: EMBAPA, 2006. $306 \mathrm{p}$.

FERRAZ, E. M. N. et al. Composição florística em trechos de vegetação de caatinga e brejo de altitude na região do Vale do Pajeú, Pernambuco. Revista Brasileira de Botânica, São Paulo, v. 21, n. 1, p. 7-15, 1998.

FERRAZ, J. S. F.; ALBUQUERQUE, U. P.; MEUNIER, I. M. J. Valor de uso e estrutura da vegetação lenhosa as margens do Riacho do Navio, Floresta, PE, Brasil. Acta Botanica Brasilica, Feira de Santana, v. 19, n. 1, p. 125-134, 2006.

FERREIRA JUNIOR, O. GPS TrackMaker ${ }^{\circledR}$ para Windows. Belo Horizonte: Geo Studio Technology, 2010.

GIULIETTI, A. M. et al. Plantas endêmicas da caatinga. In: SAMPAIO, E. V. S. B. et al. (Eds.). Vegetação e flora das caatingas. Recife: APNE; CNIP, 2002. p. 103-115.

GUEDES, R. S. et al. Caracterização florístico-fitossociológica do componente lenhoso de um trecho de 
caatinga no semiárido paraibano. Revista Caatinga, Mossoró, v. 25, n. 2, p. 99-108, 2012.

INSTITUTO DE PLANEJAMENTO DO CEARÁ. Perfil básico municipal. Fortaleza: IPLANCE, 2012. Disponível em: <http://www.ipece.ce.gov.br/publicacoes/perfil_basico/pbm-2012/Iguatu.pdf > Acesso em: 12 abr. 2013.

LEMOS, J. R.; MEGURO, M. Florística e fitogeografia da vegetação decidual da Estação Ecológica de Aiuaba, Ceará, Nordeste do Brasil. Revista Brasileira de Biociências, Porto Alegre, v. 8, n. 1, p. 34-43, 2010.

LEMOS, J. R.; RODAL, M. J. N. Fitossociologia do componente lenhoso de um trecho da vegetação de caatinga no Parque Nacional Serra da Capivara, Piaú, Brasil. Acta Botanica Brasílica, Feira de Santana, v. 16, n. 1, p. 23-42, 2002.

LEWIS, G. P. Caesalpinia: a revision of the poincionella-erythrostemon group. Kew: Royal Botanical Gardens, 1998. 233 p.

MAGURRAN, A. E. Diversidad ecológica y su medición. Madri: Ediciones Vedrà, 1989. 200 p.

MARANGON, G. P. et al. Estrutura e padrão espacial da vegetação em uma área de caatinga. Floresta, Curitiba, v. 43, n. 1, p. 83-92, 2013.

MUELLER-DOMBOIS, D.; ELLENBERG, H. Aims and methods of vegetation ecology. New York: John Wiley \& Sons, 1974. 547 p.

OLIVEIRA, P. T. B. et al. Florística e fitossociologia de quatro remanescentes vegetacionais em áreas de serra no cariri paraibano. Revista Caatinga, Mossoró, v. 22, n. 4, p. 169-178, 2009.

PEGADO, C. M. A. et al. Efeitos da invasão biológica de algaroba - Prosopis juliflora (Sw.) DC. sobre a composição e a estrutura do estrato arbustivo-arbóreo da caatinga no Município de Monteiro, PB, Brasil. Acta Botânica Brasílica, Feira de Santana, v. 4, n. 20, p. 887-898, 2006.

PEREIRA JÚNIOR, L. R.; ANDRADE, A. P.; ARAÚJO, K. D. Composição florística e fitossociológica de um fragmento de caatinga em Monteiro, PB. Revista Holos, Natal, v. 6, n. 28, p. 76-87, 2012.

PESSOA, M. F. et al. Estudo da cobertura vegetal em ambientes da caatinga com diferentes formas de manejo no assentamento Moacir Lucena, Apodi - RN. Revista Caatinga, Mossoró, v. 21, n. 3, p. 40-48, 2008.

PINHEIRO, K.; ALVES, M. Espécies arbóreas de uma área de Caatinga no sertão de Pernambuco, Brasil: dados preliminares. Revista Brasileira de Biociências, Porto Alegre, v. 5, n. 52, p. 426-428, 2007.

QUEIROZ, J. A. et al. Análise da estrutura fitossociológica da Serra do Monte, Boqueirão, Paraíba. Revista de Biologia e Ciências da Terra, Campina Grande, v. 6, n. 1, p. 251-259, 2006.

RAMALHO, C. I. et al. Flora arbóreo-arbustiva em áreas de Caatinga no semi-árido baiano, Brasil. Revista Caatinga, Mossoró, v. 22, n. 3, p. 182-190, 2009.

RODAL, M. J. N.; SAMPAIO, E. V. S. B.; FIGUEIREDO, M. A. Manual sobre métodos de estudos florístico e fitossociológico: ecossistema caatinga. Brasília: SBB, 2013. 24 p. Disponível em: <http:/ /www. botanica.org.br/ebook>. Acesso em: 12 nov. 2013.

RODAL, M. J. N.; COSTA, K. C. C.; SILVA, A. C. B. L. Estrutura da vegetação caducifólia espinhosa (Caatinga) de uma área do sertão central de Pernambuco. Hoehnea, São Paulo, v. 35, n. 2, p. 209-217, 2008.

RODAL, M. J. N.; MARTINS, F. R.; SAMPAIO, E. V. S. B. Levantamento quantitativo das plantas lenhosas em trechos de vegetação de caatinga em Pernambuco. Revista Caatinga, Mossoró, v. 21, n. 3, p. 192-205, 2008.

RODAL, M. J. N.; NASCIMENTO, L. M. The arboreal component of a dry forest in Northeastern Brazil. Revista Brasileira de Biologia, São Carlos, v. 66, n. 2, p. 479-492, 2006.

SANTANA, J. A. S.; SOUTO, J. S. Diversidade e Estrutura Fitossociológica da Caatinga na Estação Ecológica do Seridó-RN. Revista de Biologia e Ciências da Terra, Campina Grande, v. 6, n. 2, p. 232242, 2006.

SANTOS, D. S.; JERÔNIMO, C. E. M. Levantamento florístico do município de Pedra Preta-RN: subsídios para empreendimentos futuros. Revista Eletrônica em Gestão, Educação e Tecnologia Ambiental, Santa Maria, v. 15, n. 15, p. 2925- 2934, 2013.

SOUZA, G. F.; MEDEIROS, J. F. Fitossociologia e florística em áreas de caatinga na microbacia hidrográfica do Riacho Cajazeiras- RN. Geotemas, Pau dos Ferros, v. 3, n. 1, p. 161-176, 2013. 
SOUZA, J. A. N.; RODAL, M. J. N. Levantamento florístico em trecho de vegetação ripária de caatinga no Rio Pajeú, Floresta / Pernambuco - Brasil. Revista Caatinga, Mossoró, v. 23, n. 4, p. 54-62, 2010.

TOLEDO, K. Apenas 7,5\% da Caatinga está protegida. São Paulo: Agencia FAPESP, 2013. Disponível em: <http://agencia.fapesp.br/17460>.Acesso em: 20 jun. 2013.

VELOSO, H. P.; RANGEL-FILHO, A. L. R.; LIMA, J. C. A. Classificação da vegetação brasileira, adaptada a um sistema universal. Rio de Janeiro: IBGE, 1991. 112 p. 